\title{
Health Education Materials for Arab Patients: Content and Design Preferences
}

\author{
Muhammad Jawad Hashim Halla Mustafa Amani Obaid Al Abdouli \\ Reem Ahmed Abdulla Al Salama Mohamed AlQahtani \\ Salama Abdulbasit Almajed Shamma Saif Alzaabi Mariam Salem Alkaabi \\ Faculty of Medicine and Health Sciences, United Arab Emirates University, Al-Ain, United Arab Emirates
}

\section{Key Words}

Health education · Arabic world · Patient education handout color trifold brochures illustrated with pictures and not clipart and written in Arabic using the Simplified Arabic font in 16-point size.

Copyright $\odot 2013$ S. Karger AG, Basel

\begin{abstract}
Objective: To investigate the content and design preferences of printed health education leaflets among Arab patients.

Materials and Methods: A survey questionnaire to 17 subjects (women: $n=8$, men: $n=9$; age range $17-70$ years) and three focus-group discussions (total 16 participants) were used to assess preferences by showing samples of Arabic health education materials. The questionnaire was administered by interviewers. For the focus-group discussions, the sessions were conducted by a trained interviewer, audio recorded and analyzed thematically. The subjects' educational level was from no formal schooling to university level. $\boldsymbol{R e}$ sults: In survey component, all patients preferred photographs over clipart. Typeface ('font') preferences were for Simplified Arabic in 8 subjects (47\%) and Mudir MT in 7 (41\%); the 16-point font size was favored by 14 (82\%) patients. In the three focus-group discussions, themes that participants expressed included use of standard Arabic with local dialects, short sentences, and culturally appropriate advice with practical and quantifiable examples. Conclusions: The participants preferred health education materials to be
\end{abstract}

\section{Introduction}

The design of health education materials requires consideration of several factors including content, literacy demand, numeracy, graphics, layout and typography [1]. A significantly high proportion of the studies in the field are concerned with readability of written information [2] while scant attention is paid to the format and layout. As noted by Pander Maat and Lentz [3], redesigning or reformatting the layout can substantially improve comprehension and information localization in patient information leaflets. Emerging guidelines on the formatting of drug information leaflets are beginning to set minimum standards on information content and presentation. Unfortunately, these guidelines are often ignored as observed in one of the few studies on typography in printed drug information sheets [4].

In the Arab region, the lack of development of clear standards for layout [5] and typography for Arabic print-

\begin{tabular}{ll}
\hline KARGER & $\begin{array}{l}\text { ○ 2013 S. Karger AG, Basel } \\
1011-7571 / 13 / 0224-0411 \$ 38.00 / 0 \quad \text { Karger }\end{array}$ \\
E-Mail karger@karger.com & $\begin{array}{l}\text { This is an Open Access article licensed under the terms of the } \\
\text { www.karger.com/mpp }\end{array}$ \\
$\begin{array}{l}\text { Creative Commons Attribution-NonCommercial 3.0 Un- } \\
\text { ported license (CC BY-NC) (www.karger.com/OA-license), } \\
\text { applicable to the online version of the article only. Distribu- } \\
\text { tion permitted for non-commercial purposes only. }\end{array}$
\end{tabular}

Muhammad Jawad Hashim, MD Department of Family Medicine Faculty of Medicine and Health Sciences UAE University, PO Box 17666, Al Ain (United Arab Emirates) E-Mail jhashim@uaeu.ac.ae 
Table 1. Demographic characteristics of study participants

\begin{tabular}{lc}
\hline Characteristic & Participants \\
\hline Questionnaire survey participants & \\
Age & \\
$\quad \leq 40$ years & $6(35)$ \\
$\quad>40$ years & $11(65)$ \\
Gender: women & $8(47)$ \\
Ethnicity: Arab & $17(100)$ \\
Years of schooling & \\
$\quad \leq 10$ & $9(53)$ \\
$\quad>10$ & $8(47)$ \\
Acknowledges need for assistance & \\
$\quad$ in filling out written forms & $12(71)$ \\
Focus-group discussion participants & \\
$\quad$ First focus-group & 5 \\
$\quad$ Second focus-group & 6 \\
$\quad$ Third focus-group & 5 \\
$\quad$ Gender: women & $16(100)$ \\
$\quad$ Age range, years & $25-45$ \\
\hline
\end{tabular}

Number and percent of participants are given.

ing makes development of health materials a daunting task. Traditionally, Arab society has followed an oral culture and regional health promotion models have preferred verbal information and audiovisual presentations $[6,7]$. However, there is an increasing need for culturally appropriate well-designed health education materials for the Middle East region [8]. Gaps in knowledge, awareness and practices have been found in regional surveys on dental care [9], diabetes self-care [10] and cervical cancer screening [11]. We did not find any published research for regional health education although attempts at developing a theoretical model have been made [12]. This study was conducted to delineate the preferred format and content style of printed Arabic health education materials.

\section{Materials and Methods}

A mixed methods approach was used by combining quantitative (an interviewer-administered questionnaire survey) and qualitative (focus-group discussions) methods. Mixed methods converge information in exploring new research areas, providing a framework for triangulation and data transformation models [13]. The study was approved by the Hospital Institutional Committee as well as medical research Ethics Committee and written informed consent was obtained from participants.
Questionnaire Survey

Seventeen Arabic-speaking subjects were recruited for this component of our study; 8 were women and 9 men; ages ranged from 17 to 70 years. Subjects were recruited from clinic waiting areas at Tawam Hospital, a public hospital in the city of Al Ain, United Arab Emirates. Inclusion criteria were age more than or equal to 16 years, no apparent visual, hearing or mental impairment and willingness to consent for research. Healthcare workers were excluded from participating.

An interviewer-administered 8-item limited response questionnaire was used to measure the design preferences in four domains: print format, typeface (font), numerals and images. The form was pilot-tested initially for acceptability and any practical issues; however, no major revisions were needed. The single preferred choice for each item by a participant was recorded by the interviewers (A.O.A.A., R.A.A.A., S.M.A., S.A.M.A., S.S.A., M.S.A.), who were not blinded to the participants' demographics.

\section{Focus-Group Discussions}

Three focus-group discussion sessions were conducted at different locations: university administration: 5 females, ages 25-30 years; city zoo staff: 6 females, ages $25-45$ years, and residents of a local community: 5 females, ages $22-45$ years. A structured moderator's guide was first developed that included a checklist and standard opening questions for the focus-group discussions. An experienced moderator (H.M.) led three sessions which were audio recorded. Saturation was reached when previously discussed issues were repeated and the sessions stopped. The session notes and the audio recordings were carefully analyzed (without transcribing) using a thematic approach. Common themes were drawn from the discussions and related to the variables in the sample population.

\section{Sample Size Calculation}

We were not able to estimate a sample size due to lack of estimates or published research in this area. A post hoc power analysis revealed that with 17 subjects, an effect size of 0.52 (from one of the observed results), and an alpha level of 0.05 , the power achieved was 99\% (GPower software, F. Faul, University of Kiel, Germany).

\section{Statistical Analysis}

Using SPSS version 19 (Chicago, Ill., USA), we applied onesample non-parametric tests (binomial and $\chi^{2}$ tests) to assess whether the response options were equally distributed (null hypothesis: all options are equally preferred).

\section{Results}

\section{Questionnaire Survey}

The mean age of the study participants was $47.4 \pm 14.3$ years and the mean duration of completed education was $9.5 \pm 5.3$ years (table 1 ). Participants preferred the Simplified Arabic typeface in the 16-point size as well as Arabic text over bilingual English-Arabic format. Photographs were overwhelmingly chosen over clipart images. A trifold brochure was more attractive to the participants compared to an unfolded sheet of paper. 
Table 2. Arab patients' preferences for design of health education leaflets

\begin{tabular}{|c|c|c|}
\hline Design preference & Participants & $\mathrm{p}$ value \\
\hline Typeface (font) & & 0.018 \\
\hline Simplified Arabic & $8(47)$ & \\
\hline Mudir MT & $7(41)$ & \\
\hline Akhbar MT & $1(6)$ & \\
\hline Traditional Arabic & $1(6)$ & \\
\hline Arabic typesetting & $0(0)$ & \\
\hline Font size & & $<0.0001$ \\
\hline 16-point & $14(82)$ & \\
\hline 14-point & $1(6)$ & \\
\hline 12-point & $2(12)$ & \\
\hline 10-point & $0(0)$ & \\
\hline \multicolumn{3}{|l|}{ Images } \\
\hline Photographs & $17(100)$ & \\
\hline Clipart & $0(0)$ & \\
\hline Number format & & 0.003 \\
\hline Both & $12(71)$ & \\
\hline Eastern Arabic $(\mid r r \ldots)$ & $4(24)$ & \\
\hline Indo-Arabic $\left(\begin{array}{lll}1 & 2 & 3 \ldots\end{array}\right)$ & $1(6)$ & \\
\hline Language & & 0.049 \\
\hline Arabic only & $13(76)$ & \\
\hline Arabic with English & $4(24)$ & \\
\hline Leaflet format & & $<0.0001$ \\
\hline Trifold brochure & $16(94)$ & \\
\hline Plain (not folded) sheet & $1(6)$ & \\
\hline
\end{tabular}

Number and percent of participants are given; $\chi^{2} p$ value of less than 0.05 indicates that the choices are unlikely to be equally preferred.

\section{Focus-Group Discussions}

The following themes were generated on analysis of the audio recordings and notes: health education advice should be based on Arab customs and practices, as one participant indicated that 'early morning walks for women were not practical'. Practical examples for each advice were requested such as stating specific self-rewards for achieving personal goals. Participants asked that dietary advice should recommend locally available foods while avoiding referring to 'exotic fruits' and unfamiliar cuisine. They suggested that time durations be specified in minutes or hours instead of vague references. Images of high quality and large size were felt to be needed, especially comparative photos, for example, 'small and large meal portion sizes'. Attractive titles for leaflets that catch attention, for instance, with a phrase or a question, should be utilized. Participants also recommended using shorter sentences, even minimal text complemented by many photographs. Standard Arabic with local dialects in parentheses should be printed with diacritical marks (hara$k a h)$ for wider usage.

\section{Discussion}

\section{Content}

In this study on the preferences of Arab patients for printed health education materials, the use of culturally appropriate and acceptable health advice was felt to be essential. Recommendations by our study subjects such as the need for short sentences, clear examples, comparisons and quantified information corroborate a recent systematic review of health education interventions [14]. These may be cross-cultural preferences, although some of our findings are applicable to Arab communities only, such as the use of standard Arabic as opposed to regional dialects and the placement of diacritical marks on printed words. However, as Sheridan et al. [14] point out, although simplified text has a beneficial effect on patients with limited literacy, the use of media such as pictures has had conflicting results on comprehension in randomized controlled trials of health education materials.

\section{Design}

Based on our findings, the typeface preference seems to be dictated by clarity and boldness as both Simplified Arabic and Mudir MT are heavy naksh-style fonts as opposed to the finer nastaleeq printing. The predilection for the largest font size (16-point) suggests the need for a larger-sized body text in printed Arabic than for Roman (English) letters at similar reading distances. This finding needs further confirmation in controlled reading comprehension tests. Practical aspects of the regional culture and environment require reflection and scrutiny when developing health education for Arab audiences [8]. Images from local settings resonate with this group of patients more than generic clipart, which are often based on Western images.

Limitations of our study include lack of a pre-validated questionnaire. The sample size was small and this should be considered a pilot study; however, additional subjects may not have led to substantially different findings. Saturation of themes as well as triangulation was achieved with cross-confirmation of observations from the qualitative and quantitative arms. Further studies should assess comprehension and clinical outcomes in this population. 


\section{Conclusions}

This study has provided content and design guidance to authors of health education materials for Arabicspeaking patients. Health education materials targeted to Arab-speaking patients should preferably be culturally appropriate using local examples, illustrated with pictures but not generic clipart and written in Arabic preferably using the Simplified Arabic font in 16-point size.

\section{Acknowledgements}

We would like to thank all the participants of our study. This research was supported by a grant from the Emirates Foundation (EF2009/217).

\section{References}

1 Helitzer D, Hollis C, Cotner J, Oestreicher N: Health literacy demands of written health information materials: an assessment of cervical cancer prevention materials. Cancer Control 2009;16:70-78.

2 Aldridge MD: Writing and designing readable patient education materials. Nephrol Nurs J 2004;31:373-377.

3 Pander Maat H, Lentz L: Improving the usability of patient information leaflets. Patient Educ Couns 2010;80:113-119.

4 Chubaty A, Sadowski CA, Carrie AG: Typeface legibility of patient information leaflets intended for community-dwelling seniors. Age Ageing 2009;38:441-447.
5 Nasir LS, Abdul-haq AK: Caring for Arab Patients: A Biopsychosocial Approach. Milton Keynes, Radcliffe, 2008.

6 Elkamel F: The use of television series in health education. Health Educ Res 1995;10: 225-232.

7 el-Katsha S, Watts S: A model for health education. World Health Forum 1994;15:29-33.

8 Nasir LS, Nasir AK: Introducing Arabic language patient education materials in Jordan. Patient Educ Couns 2006;60:142-145.

-9 Ashkanani F, Al-Sane M: Knowledge, attitudes and practices of caregivers in relation to oral health of preschool children. Med Princ Pract 2012, E-pub ahead of print.

10 Awad A, Dalle H, Enlund H: Diabetic patients' knowledge of therapeutic goals in $\mathrm{Ku}$ wait. Med Princ Pract 2011;20:118-123.
Al Sairafi M, Mohamed FA: Knowledge, attitudes, and practice related to cervical cancer screening among Kuwaiti women. Med Princ Pract 2009; 18:35-42.

12 Kulwicki A: Patient education; in Nasir LS Abdul-haq AK (eds): Caring for Arab Patients: A Biopsychosocial Approach. Milton Keynes, Radcliffe, 2008, pp 235-246.

13 Creswell JW, Fetters MD, Ivankova NV: Designing a mixed methods study In primary care. Ann Fam Med 2004;2:7-12.

14 Sheridan SL, Halpern DJ, Viera AJ, Berkman ND, Donahue KE, Crotty K: Interventions for individuals with low health literacy: a systematic review. J Health Commun 2011;16:30-54. 